\title{
SwissDRG:
}

\section{un enjeu majeur pour la FMH}

Que les nostalgiques de l'opposition se rendent à l'évidence: la base légale a été scellée, les manœuvres dilatoires déjouées, SwissDRG se fera. Etant donné que dans ce type de structure tarifaire, rien ne peut se construire sans la contribution des médecins, il est essentiel que la FMH y joue un rôle clé et s'y affirme en tant que leader incontournable. La rétribution des prestations hospitalières sur la base de forfaits va remodeler les conditions de travail de la moitié d'entre nous: cette perspective justifie le développement d'une stratégie élaborée et l'attribution des moyens nécessaires à son application.

Au niveau du Comité central de la FMH, la création d'un dicastère SwissDRG a été réalisée depuis déjà deux ans. Face aux autres acteurs du système de santé, nous avons réussi à devenir partenaire à part entière de la nouvelle société SwissDRG SA. Notre engagement politique a permis certains revirements bienvenus, en particulier le maintien du financement de la formation postgraduée dans le calcul des forfaits hospitaliers. Cependant, il convient de veiller au grain: les nouvelles ordonnances en application des modifications de la LAMal sont actuellement en consultation et doivent tenir compte de nos objectifs.

A l'échelon opératif, la création d'une équipe de travail à plein temps au sein de la FMH est maintenant indispensable. Ce groupe aura pour tâche de coordonner toutes les activités de notre fédération concernant SwissDRG et d'être le répondant de nos membres ainsi que de nos sociétés à ce sujet. A l'aide d'experts allemands de haut niveau travaillant sur mandat, il profitera de l'expérience de nos voisins et évitera de reproduire certaines erreurs dans le système suisse. Pour faire face à des pics d'activité liés au cycle des demandes d'adaptation, il continuera à recourir ponctuellement aux capacités locales, présentes dans certains de nos grands hôpitaux.

2008 et 2009 seront décisifs. Un remaniement important de notre manuel de codification CHOP est envisagé; il dépendra directement de la qualité des propositions qui seront faites par les sociétés de discipline cet été. D'autre part, un budget significatif a été prévu dès l'année prochaine pour que la FMH puisse garder le premier rôle aussi bien dans l'élaboration que dans l'entretien du système.

SwissDRG est un projet dynamique, la révision de la structure tarifaire survenant chaque année sur la base des propositions des partenaires. On le voit, notre engagement sera nécessaire à long terme et il faut s'en donner les moyens. Avec le soutien de chacun, le dicastère SwissDRG est prêt à relever ce défi.

Dr Pierre-François Cuénoud, membre du Comité central de la FMH, responsable du domaine SwissDRG

Pour plus d'informations sur SwissDRG, veuillez consulter le site www.fmh.ch $\rightarrow$ Nos prestations. 